\title{
Numerical Simulations of Turbulent Combustion
}

\author{
Andrei N. Lipatnikov \\ Department of Mechanics and Maritime Sciences, Chalmers University of Technology, 41296 Gothenburg, \\ Sweden; andrei.lipatnikov@chalmerse.se
}

Received: 1 February 2020; Accepted: 5 February 2020; Published: 10 February 2020

Turbulent burning of gaseous fuels is widely used for energy conversion in stationary power generation, e.g., gas turbines, land transportation, e.g., piston engines, and aviation, e.g., aero-engine afterburners. Nevertheless, fundamental understanding of turbulent combustion is still limited, because it is a highly non-linear and multiscale process that involves various local phenomena and thousands (e.g., for gasoline-air mixtures) of chemical reactions between hundreds of species, including a number of reactions that control emissions from flames. Therefore, there is a strong need for elaborating high fidelity, advanced numerical models and methods that (i) will catch complex combustion chemistry and the governing physical mechanisms of flame-turbulence interaction and, consequently, (ii) will make turbulent combustion computations an efficient predictive tool for applied research. In particular, such computations are required to facilitate development of a new generation of ultra clean and highly efficient internal combustion engines that will allow the society to properly respond to current environmental and efficiency challenges. The goal of this special issue is to provide a forum for recent developments in such numerical models and methods. The special issue contains papers aimed at (i) developing and validating high fidelity models and efficient numerical methods for Computational Fluid Dynamics research into turbulent, complex-chemistry combustion in laboratory burners and in engines or (ii) improving fundamental understanding of flame-turbulence interaction by analyzing data obtained in unsteady multi-dimensional numerical simulations.

Khan et al. [1] report results of a joint experimental and numerical study of chemical processes that cause autoignition of a fuel-air mixture and, in particular, knock in Spark Ignition engines. More specifically, ignition delay times computed invoking three reduced (semi-detailed) chemical mechanisms for different gasoline surrogates are compared with timing of knock onset measured for a wide range of temperatures and pressures. Obtained results indicate that the studied chemical mechanisms and surrogate properties can feasibly be used in the calculation of gasoline autoignition in a Spark Ignition engine, with the computed ignition delay time being sensitive to the choice of a mechanism and/or surrogate.

Endres and Sattelmayer [2] present results of large eddy simulations performed by allowing for complex combustion chemistry at various pressures. The goal of the study is to numerically explore boundary layer flashback in a confined combustion chamber when burning hydrogen-air mixtures. Results show that while the turbulent flame speed at conditions close to flashback decreases with increasing pressure, the flashback propensity is increased by the pressure. This finding indicates that a single quantity such as the turbulent flame speed is a poor indicator for the onset of boundary layer flashback. The flashback is a complex process affected by the flame speed, the flame thickness, the quenching distance, and the local separation zone size. Moreover, the computed results show that the underlying assumptions of the boundary layer theory are not satisfied under conditions of the study. For instance, application of one-dimensional pressure approximations results in overestimating the pressure increase ahead of the flame.

To perform large eddy simulations of turbulent burning of ethanol sprays, Filho et al. [3] develop a modeling strategy that allows for complex combustion chemistry by combining Flamelet Generated Manifolds (GFM) and Artificially Thickened Flame (ATF) approach extended by the authors to take 
into account enthalpy variations due to evaporative cooling effects. Ethanol droplets are tracked using an Euler-Lagrangian approach and applying an evaporation model to allow for the inter-phase non-equilibrium. Numerical results are validated using experimental data obtained from flame EtF5 of the Sydney diluted spray flame burner. Moreover, a parametric numerical study is performed to assess magnitudes of effects due to evaporation cooling and wrinkling of flame surface by turbulent eddies, with the latter effect being of more importance.

Heinrich et al. [4] apply large eddy simulation to study another problem, i.e., flame-wall interaction, which can promote pollutant formations and increase heat losses, thus, lowing efficiency of an internal combustion engine. Similar to Ref. [3], complex combustion chemistry and flame-turbulence interaction are taken into account adopting the FGM and ATF approaches, respectively. The numerical model is validated using experimental data on sidewall quenching of turbulent flames, obtained recently in Darmstadt. The validation study shows that the adapted numerical approach can handle sidewall quenching of turbulent flames. Moreover, in the paper, the computed instantaneous 3D fields are analyzed and three different scenarios are revealed. These are: an upstream, a downstream and a jump-like upstream movement of the flame. In the third case, the flame behaves locally like a head-on quenching flame and the highest heat fluxes are calculated.

Alqallaf et al. [5] analyze direct numerical simulation data obtained from expanding, statistically spherical turbulent premixed flames characterized by three different Lewis numbers, i.e., Le $=0.8$, 1.0, and 1.2, with all other things being equal. By processing the data, various terms in the transport equation for the local curvature of the instantaneous flame surface are evaluated and terms due to curl of vorticity and normal strain rate gradients are found to play the most important roles in the studied transport equation in all three cases. In the case of Le $=0.8$, the net contribution of the considered terms acts to augment turbulence-induced wrinkles on the flame surface. In two other cases of Le $=1.0$ and 1.2, flame propagation tends to smoothen the flame surface. These findings shed a new light on the influence of the Lewis number on turbulent burning rate.

$\mathrm{Yu}$ and Lipatnikov [6] compare direct numerical simulation data computed by studying two model problems relevant to premixed turbulent combustion. These are (i) motion of a self-propagating interface in a constant-density turbulence and (ii) propagation of a reaction wave of a finite thickness in a constant-density turbulence. Both data sets are obtained from statistically the same turbulence. In the former case, the computed mean speed of the interface is proportional to the rms turbulent velocity $\mathrm{u}^{\prime}$, whereas the dependence of the mean wave speed on $\mathrm{u}^{\prime}$ shows bending, which is more pronounced in the case of a higher diffusivity of the reactant, i.e., a larger local wave thickness. Analysis of the data indicates that the bending effect is controlled by a decrease in the rate of an increase in the reaction-zone-surface area with increasing $\mathrm{u}^{\prime}$. This decrease stems from inefficiency of small-scale turbulent eddies in wrinkling the reaction-zone surface, because such small-scale wrinkles characterized by a high local curvature are efficiently smoothed out by molecular diffusion within the reaction wave.

Lipatnikov et al. [7] suggest new closure relations for turbulent scalar fluxes of flame surface density and scalar dissipation rate in the corresponding transport equations. These closure relations are validated by analyzing direct numerical simulation data obtained from three statistically stationary, one-dimensional, planar, weakly turbulent premixed flames characterized by three different density ratios. The models predict the fluxes reasonably well without using any tuning parameter and can yield both gradient and countergradient fluxes in different zones of the mean flame brushes, with the zone sizes depending on the density ratio.

I thank all of the authors for submitting their manuscripts for this special issue. I also thank all of the reviewers for their time and valuable comments increasing the quality of the published papers.

Conflicts of Interest: The author declares no conflict of interest. 


\section{References}

1. Khan, A.F.; Roberts, P.J.; Burluka, A.A. Modelling of self-ignition in Spark-Ignition engine using reduced chemical kinetics for gasoline surrogates. Fluids 2019, 4, 157. [CrossRef]

2. Endres, A.; Sattelmayer, T. Numerical investigation of pressure influence on the confined turbulent boundary layer flashback process. Fluids 2019, 4, 146. [CrossRef]

3. Filho, F.L.S.; Dressler, L.; Hosseinzadeh, A.; Sadiki, A.; Filho, G.C.K. Investigations of evaporative cooling and turbulence flame interaction modeling in ethanol turbulent spray combustion using tabulated chemistry. Fluids 2019, 4, 187. [CrossRef]

4. Heinrich, A.; Kuenne, G.; Ganter, S.; Hasse, C.; Janicka, J. Investigation of the turbulent near wall flame behavior for a sidewall quenching burner by means of a large eddy simulation and tabulated chemistry. Fluids 2018, 3, 65. [CrossRef]

5. Alqallaf, A.; Klein, M.; Chakraborty, N. Effects of Lewis number on the evolution of curvature in spherically expanding turbulent premixed flames. Fluids 2019, 4, 12. [CrossRef]

6. Yu, R.; Lipatnikov, A.N. DNS Study of the bending effect due to smoothing mechanism. Fluids 2019, 4, 31. [CrossRef]

7. Lipatnikov, A.N.; Nishiki, S.; Hasegawa, T. Closure relations for fluxes of flame surface density and scalar dissipation rate in turbulent premixed flames. Fluids 2019, 4, 43. [CrossRef]

(C) 2020 by the author. Licensee MDPI, Basel, Switzerland. This article is an open access article distributed under the terms and conditions of the Creative Commons Attribution (CC BY) license (http://creativecommons.org/licenses/by/4.0/). 